\title{
Posterior Ligamentous Complex Reconstruction for Cervical Dislocation and Instability in Patients without Vertebral Fracture with Artificial Ligaments: A Technical Note
}

\section{Chengyi Huang}

West China Hospital of Medicine: Sichuan University West China Hospital

\section{Beiyu Wang}

West China School of Medicine: Sichuan University West China Hospital

Hao Liu (D liuhaohxyy@163.com)

West China School of Medicine: Sichuan University West China Hospital

Tingkui Wu

West China Hospital of Medicine: Sichuan University West China Hospital

Kangkang Huang

West China School of Medicine: Sichuan University West China Hospital

Junbo He

West China School of Medicine: Sichuan University West China Hospital

Haimiti Abudouaini

West China Hospital of Medicine: Sichuan University West China Hospital

Chen Ding

West China Hospital of Medicine: Sichuan University West China Hospital

Hua Chen

West China School of Medicine: Sichuan University West China Hospital

\section{Technical advance}

Keywords: Cervical vertebra, Posterior ligamentous complex, Injury, Non-fusion, Reconstruction

Posted Date: November 13th, 2020

DOI: https://doi.org/10.21203/rs.3.rs-103566/v1

License: (1) This work is licensed under a Creative Commons Attribution 4.0 International License.

Read Full License 


\section{Abstract}

Introduction: The posterior ligamentous complex (PLC) offers restraints to deformation in a complex and interconnected manner. The stability and range of motion (ROM) of the posterior cervical spine is greatly restricted when the PLC is damaged, even without bone disruptions. We describe a novel surgical technique, its advantages, and the postoperative condition of the first patient to use artificial ligament in a reconstruction surgery of PLC injuries in patients without lower cervical vertebral fracture. This technique aimed to fully retain the mobility of the injured segment based on spinal stability and apply dynamic reconstruction in patients' treatment.

Method: We present a detailed description of the reconstruction surgery with artificial ligaments performed on C4-6 PLC injuries in a 27-year-old male presented with neck pain and restricted movement following a high fall injury accompanied by impaired movement of limbs.

Results: Immediately postsurgery, the patient's neck pain and quadriparesis had been improved. The spinal canal decompression and cervical spine sequence results were satisfactory, the facet joint face was in a good position, and the spinous process spacing returned to normal. After three months of rehabilitation, the patient reported improvement of symptoms and the physical and imaging examination showed a significant improvement in the patient's condition. The patient's neck mobility motor function had improved further.

Conclusion: The present data demonstrate that the novel technique for reconstruction of PLC injury is feasible and safe. However, familiarity with cervical anatomy and adequate experience in lateral mass screws placement during surgery is crucial for this procedure. Therefore, a highly experienced cervical surgery team is recommended to perform the surgery.

\section{Introduction}

Posterior ligamentous complex (PLC), also called the posterior tension band, consists of the supraspinous ligament (SSL), interspinous ligament (ISL), ligamentum flavum (LF), and facet joint capsules (FJC) [1, 2]. The integrity of this complex prevents excessive flexion, rotation, translation, and distraction of the spine. These structures offer restraints to deformation in a complex and interconnected manner. The stability and range of motion (ROM) of the posterior cervical spine is greatly restricted when the PLC is damaged, even without bone disruptions. Clinical symptoms mainly manifest as instability of the cervical spine and excessive motion, especially in flexion [3]. The treatment options for patients with this type of ligament injury can be categorized as either conservative treatment or surgical techniques involving fusion to prevent progressive deformity and continuous spinal cord compression due to the loss of tension of the posterior ligament [2]. Fusion surgery has been performed since the 1910s and is considered the most reliable approach. Short-segment posterior pedicle screw instrumentation has been commonly used in thoracolumbar flexion-distraction injury due to the significant effect in the reconstruction of the posterior tension band and restoration of the normal sagittal contour [4-5]. 
However, spinal fusion is not suitable for treating patients with this type of ligament structural damage without fracture, especially for the cervical spine [6-7]. Stability reconstruction of the cervical spine can cause loss of motion in fusion segments, which increases the ROM in the adjacent segments compensatorily. Previous studies based on mechanical tests have shown that spinal fixation changes movement patterns in the adjacent segment. This increases fixation stiffness and adjacent segment displacement [8]. Inevitable degeneration of adjacent segments has been confirmed during long-term follow-up [9-12].

Non-fusion techniques are new methods that can reliably rebuild stability while retaining a certain degree of motion. In terms of stability, as the stiffness of the internal system of the spine decreases properly due to the replacement of posterior pedicle screw instrumentation, the effect of stress shelter decreases, while the ROM increases appropriately. This can improve nutrition metabolization and pressure in the adjacent disk and prevent adjacent segment mobility and increases in the joint load [13-15]. Therefore, for the lower cervical spine injury without bony structural damage, it is desirable to fully retain the mobility of the injured segment based on spinal stability. Dynamic reconstruction is suggested for these patients.

Surgical reconstruction of knee cruciate ligament with bioderived tendon or artificial materials has been in use for years. Tissue-engineering techniques using bioderived tendon provide potential alternatives for recreating suitable biocompatible fixation materials [16-18]. However, similar to knee ligament rupture, some patients with lower cervical spine injuries have only posterior ligament complex rupture rather than bone structure damage. In addition, the cervical spine is not the main load-bearing structure. If there are no anterior and posterior column bone structural fractures, theoretically it may also be possible to use artificial ligament to rebuild the PLC by longitudinally connecting spinous processes and lateral cervical masses for cervical dynamic stability reconstruction.

A biomechanical analysis of bilateral facet joint stabilization using bioderived tendon in goats was conducted. The results showed that this novel non-fusion posterior cervical reconstruction method significantly limited excessive flexion motion and maintained adequate stability, proving the feasibility and effectiveness of this technique [3]. We report a novel surgical technique, its advantages, and the postoperative condition of the first patient to use artificial ligaments in a reconstruction surgery of PLC injuries in patients without structural fractures of the lower cervical spine.

\section{Case}

The patient, a 27-year-old male, suffering from neck pain and restricted movement following a high fall injury accompanied by impaired movement of limbs was admitted to our department by the emergency department. Physical examination showed the patient suffered from neck tenderness and tapping pain, accompanied by decreased skin sensation below the shoulder level. Upper extremity (UE) and lower extremity $(L E)$ motor grades $(0-5)$ were evaluated before the surgery (Table 1$)$. There were severe $(2 / 5)$ and moderate (3/5) weaknesses in the left and right UE, respectively. Meanwhile, severe (0/5) and mild $(4 / 5)$ weaknesses existed in the left and right LE, respectively. The preoperative Japan Orthopaedic 
Association (JOA) score was 1, Neck Disability Index (NDI) was 40, neck Visual Analog Scale (VAS) was 6, arm VAS was 4 [19-20]. As for the International Standards for Neurological Classification of Spinal Cord Injury (ISNCSCI) score, the UE motor score (UEMS) was 19, LE motor score (LEMS) was 20, lighttouch (LT) score was 76, and pinprick (PP) score was 75. The ASIA Impairment Scale (AIS) was grade C [21-22]. The functional spinal unit (FSU) Cobb angle was $-7.28^{\circ}$ and the $C 2-7$ Cobb angle was $-7.69^{\circ}$ as measured on preoperative lateral X-ray. Preoperative CT scans showed no structural fractures of the cervical spine.. Preoperative magnetic resonance imaging (MRI) revealed soft tissue edema around the cervical spinous process and spinal cord edema of C4-6, suggesting posterior tension band and spinal cord injury (Fig. 1).

Immediately postsurgery, the patient's neck pain and quadriparesis had been improved. Three days after the operation, there were still neuropathic complaints in the 4 limbs, but there was an improvement of the left UE (3/5), right UE (4/5), left LE (1/5), and right LE (5/5). The postoperative JOA score was 5, NDI was 23 , VAS neck pain score was 3 , and the VAS arm pain score was 1 . As for the ISNCSCI score, the UEMS was 25 , LEMS was 30 , the LT score was 86 , and the PP score was 81 . The AIS was grade D. The ROM of the treated level was $8.89^{\circ}$ and the overall ROM was $17.56^{\circ}$. The FSU Cobb angle was $-2.56^{\circ}$ and the C27 Cobb angle was $-1.29^{\circ}$. Postoperative CT scans revealed the proper location of the anchoring screws. The scan also showed that the facet joint face was in a good position and spinous process spacing had returned to normal. MRI showed satisfactory spinal canal decompression and cervical spine sequence (Fig. 2). In addition, color Doppler ultrasound showed mechanical properties and peripheral blood supply around the artificial ligament during flexion and extension (Fig. 3). The patient's neck mobility improved significantly (Fig. 2).

After three months of rehabilitation, the patient's quadriparesis, especially the left lower extremity, had been significantly improved. The muscle strength of left UE was grade 4, right UE was grade 5, left LE was grade 4 , and the right LE was grade 5 . The postoperative JOA score was 11, NDI was 12, VAS neck pain score was 0 , VAS arm pain score was 0 . As for the ISNCSCI score, the UEMS was 37 , LEMS was 45 , the LT score was 105 , and the PP score was 96 . The AIS was grade D. The patient could walk autonomously; the hand dexterity had improved significantly. Basically, he could live by himself with a stable neurological situation. The ROM of the treated level was $8.76^{\circ}$ and the overall ROM was $18.65^{\circ}$. The FSU Cobb angle was $-3.42^{\circ}$ and the $\mathrm{C} 2-7 \mathrm{Cobb}$ angle was $1.21^{\circ}$. The accuracy of pedicle screw placement was examined postoperatively using the CT scan. The X-rays showed a satisfactory cervical spine sequence. MRI demonstrated satisfactory healing of the spinal cord and back soft tissue edema; the endplates were in a normal spine alignment (Fig. 4).

Generally, the patient reported improvement of symptoms and the physical and imaging examination showed a significant improvement in the patient's condition after 3 months. The patient's neck mobility and motor function had improved further (Fig. 4).

\section{Surgical Technique}




\subsection{Posture and exposure}

First, we placed the patient in a prone position after routine sterilization. A Mayfield skull clamp frame was used to keep the head in a neutral position and moderately extend. A C-arm fluoroscope was used to confirm that the dislocated segment had been reset. A longitudinal posterior midline incision was made and the lamina and bilateral facet joints of the C4-6 cervical vertebrae were exposed by subperiosteal dissection. After the surgical exposure, it was found that the spinous process of $\mathrm{C} 4-5$ spacing was increased significantly, ISL and SSL were ruptured, and ligament fiber sequence disordered. The bilateral FJC was torn and the articular process was semi-dislocated. Interspinous ligament of C5-6 had a severe contusion and the partial facet joint had contusion and laceration. There were no fractures in the posterior spinous process, lamina, and bilateral articular processes of C4-6 (Fig. 5).

\subsection{C4-6 artificial ligament reconstruction between spinous processes}

Second, we prepared a cortical bone groove of about $4 \mathrm{~mm}$ in diameter on both sides of the base of the cervical spinous process of C4-6. Reduction forceps assist was pointed to form a bone channel with a diameter of about $4 \mathrm{~mm}$ through the bone groove. The LARS (ligament augmentation and reconstruction system) (Arc-sur-Tille, France) artificial ligament was used for reconstruction. The guidewire with the headend of the ligament was passed through the $\mathrm{C} 4$ spinous process channel, then down the opposite side through the $\mathrm{C} 5$ interspinous process channel to the same side. It was then passed further down to the $\mathrm{C} 6$ spinous process channel from the opposite side to the same side. The head and tail of the ligament were fully pulled to restore and maintain sufficient tension between the spinous processes, then the head and tail of the ligament were crossed and tied using end-to-end anastomosis tethering of the sutures (Fig. 6).

\subsection{C4-6 facet joint artificial ligament anchoring}

Third, bone channels that were parallel to the articular surfaces of the articular processes were drilled to a diameter of about $4 \mathrm{~mm}$ and a depth of about $1.5 \mathrm{~cm}$ in the middle of the C4-6 bilateral lateral masses. The headends of the two LARS artificial ligaments were respectively placed into the C4 bilateral lateral masses bone channels about $1 \mathrm{~cm}$ deep. Then two hollow squeeze screw (Arc-sur-Tille, France) with a diameter of $4.7 \mathrm{~mm}$ and a length of $1.5 \mathrm{~cm}$ was inserted into the channels to anchor the headends of the ligaments. With proper tension maintained, the middle of the ligaments was passed into the $\mathrm{C} 5$ bilateral lateral masses bone channels. Hollow squeeze screws with a diameter of $4.7 \mathrm{~mm}$ and a length of $1.5 \mathrm{~cm}$ were inserted into the channels to anchor the middle of the ligaments. Then the tail ends of the ligaments under proper tension were passed into the $\mathrm{C} 6$ bilateral lateral masses bone channels. Hollow squeeze screws with a diameter of $4.7 \mathrm{~mm}$ and a length of $1.5 \mathrm{~cm}$ were inserted into the channels to anchor the tail of the ligaments (Fig. 7). The completed state of posterior ligamentous complex reconstruction is shown in Fig. 7.

\subsection{Close the incision}


Finally, C-arm fluoroscopy was conducted to verify the locations of the anchoring screws, the recovery of the C4-6 cervical spine sequence, and determine whether the anchoring ligament tension was moderate before the wound was closed layer-by-layer.

This technical note was approved by the Medical Ethical Committee of West China Hospital, Sichuan University, China. The patient agreed to the academic publication.

\section{Discussion}

The motion of an intact cervical spinal segment is managed by the coordination of paravertebral muscles, discs, facet joints, and ligaments. Any injury to these structures can influence the function of the cervical spine, particularly in cases with PLC injury [23]. In previous spinal kinematic studies, researchers analyzed changes in the ROM and locations of the instantaneous axes of rotation of spinal segments to evaluate the biomechanical role of each ligament by sequentially cutting the ligaments [24-27]. The results of these studies indicate that SSL or ISL ligaments are the most resistant structures of the PLC to compression forces, and FJC contributes less to resistance to compression forces. Therefore, when the PLC elements are injured, a major proportion of this force shifts onto the intervertebral disc because of the loss of resistance to compression forces. Meanwhile, the cervical spine loses the restriction of the posterior ligament, leading to vertebral instability, spinal canal compression, and cervical spine sequence disorder.

A posterior element failure commonly occurs alongside an anterior structure injury [23]. Therefore, such a simple posterior tension band injury without fracture is rare. The commonly used operative treatment for cervical PLC injury includes pedicle screw and plate fixation through anterior approach, and screw-rod constructs using transfacet screws or lateral mass screws through posterior approach. To achieve the cervical spine fusion, they can also be combined to stabilize three-column damage or highly unstable cervical spine [28-35]. A posterior compression type construct and fusion is favorable for treating thoracolumbar spine PLC injury (often with fractures) due to its strong fixing ability, which is enough to withstand the gravity of the upper body [36]. However, the cervical spine has high requirements for movement and flexibility, which are inevitably limited by fusion. Besides, an increased risk of adjacent segment degeneration cannot be ignored either [37]. Conversely, the fusion treatment is less favorable for cervical PLC injuries.

Non-fusion therapy with artificial ligament reconstruction for PLC injuries on humans has not been reported. However, previous researchers have proposed some approaches similar to the one introduced in the present study. Ngo et al. used interlaminar wiring fixation looped between the spinous process base to treat patients with fracture and contralateral dislocation of the twin facet joints of the lower cervical spine [38]. This is similar to our reconstruction of the interspinous ligament using the artificial ligament with a figure " 8 " cross-tie. However, this method is essentially a fusion therapy due to the high rigidity of the wire. Moreover, the technique has certain risks; it may cause serious neurologic sequelae because the wire is passed beneath the lamina. Wang et al. conducted biomechanical test of bilateral facet joint 
stabilization using a bioderived tendon with a figure "8" cross-tie through the bone tunnels drilled through the facet joint to simulate the function of broken joint capsule ligaments. This inspired the creation of our new technology [3]. Besides, Hamoud et al. presented a new method for stabilization of a rare unstable flexion-distraction injury of the upper cervical spine in a toddler. They performed a posterior approach fixation of the spinous processes with absorbable sutures without fusion. The toddler had a good prognosis and no complications [39]. Though the unique anatomy and biomechanics of the pediatric cervical spine determine the difference between pediatric and adult injury patterns and treatment options, it still illustrates the feasibility of non-fusion technologies.

Based on previous clinical research and patient condition, we created a novel surgical technique, involving artificial ligament anchoring of the facet joints and artificial ligament reconstruction between spinous processes, to simulate the function of damaged FJC, SSL, and ISL. However, currently, this technology is only applicable to a pure rear tension band damage case (AO type B2 according to the AOS pine subaxial cervical spine injury classification system [40]). It might be more suitable for traditional fusion if combined with a fracture or severe cervical instability.

The advantages of this technique are as follows: (1) The target structure of the surgical procedure is direct and the operation procedure is clear, which improves repeatability. (2) It uses ligament-screw anchoring construct and interspinous artificial ligament reconstruction to separately simulate the FJC and the interspinous spine ligament (SSL and ISL) functions. This restores the anatomical structure and biomechanical function of the cervical spine. (3) Three parallel artificial ligaments provide sufficient tension for the cervical spine and guarantee the maintenance of stability and normal physiological curvature of the cervical spine. In addition, the figure " 8 " fixation method between the spinous processes also achieves a balance of forces on both sides of the spinous process. (4) The ligament is an elastic material that can be properly extended or contracted according to the patient's neck movement, which greatly preserves the patient's neck mobility. (5) Finally, we improved the stabilization method of bilateral facet joints in Wang et al study [3]. The bone tunnels drilled through the facet joint for the passage of bioderived tendon may cause facet joint degeneration; hence, we used lateral mass screw anchoring as an alternative.

However, our technology has some limitations. First, since this is a novel technology, only a single operation was applied. An insufficient number of cases limits the objective evaluation of surgical effect and safety. Second, since the long-term follow-up of the patient is in progress, long-term prognosis, especially the existence of unpredictable potential complications, cannot be presently addressed. Third, this technology was only applied to the PLC injury of the cervical spine. Future studies should apply the technology in the repair of the PLC injury of thoracolumbar segments or patients with minor cervical fractures, to improve the technology under various conditions. To fully understand the implications of this technique, active follow-up and evaluation of our present patient will be maintained. The technology will also be frequently applied to increase the number of cases. Further biomechanical experiments and finite element analysis with this technology are necessary for the future. 


\section{Conclusions}

In conclusion, the present data demonstrate that the novel technique for reconstruction of PLC injury may be feasible and safe. However, familiarity with cervical anatomy and adequate experience for the lateral mass screws placement during the surgery is crucial for this procedure. Therefore, this surgery should be best conducted by a highly experienced cervical surgery team.

\section{Abbreviations}

PLC: Posterior ligamentous complex

SSL: supraspinous ligament

ISL: interspinous ligament

LF: ligamentum flavum

FJC:facet joint capsules

ROM: range of motion

UE: upper extremity

LE: lower extremity

JOA: Japan Orthopaedic Association

NDI: Neck Disability Index

VAS: Visual Analog Scale

ISNCSCI: International Standards for Neurological Classification of Spinal Cord Injury

UEMS: UE motor score

LEMS: LE motor score

LT: light-touch

PP: pinprick

AIS: ASIA Impairment Scale

FSU: functional spinal unit

\section{Declarations}




\section{Ethics approval and consent to participate:}

This technical note was approved by the Medical Ethical Committee of West China Hospital, Sichuan University, China (No. 2020(640)). Written informed consent was obtained from individual or guardian participants.

\section{Consent for publication:}

Written informed consent for publication was obtained from all participants.

\section{Availability of data and materials:}

The datasets used and/or analysed during the current study are available from the corresponding author on reasonable request.

\section{Competing interests $\square$}

The authors declare that they have no competing interests.

\section{Funding:}

This study was supported by:

1.Foundation for Science \& Technology Department of Sichuan Provincial Government, China (grant no. 2020YFS0084)

2.Foundation for Science \& Technology Department of Sichuan Provincial Government, China (grant no. 2019YFQ0002)

3.1.3.5 project for disciplines of excellence-Clinical Research Incubation Project, West China Hospital, Sichuan University (grant no. 2019HXFH040)

4.Foundation for Science \& Technology Department of Sichuan Provincial Government, China (grant no. 2019YFS0135)

\section{Authors' contributions:}

$\mathrm{CH}$ was the lead investigator. $\mathrm{CH}, \mathrm{BW}$ and $\mathrm{HL}$ contributed to the conception of the new technique; $\mathrm{BW}$ and $\mathrm{HL}$ conducted the surgery; $\mathrm{CH}, \mathrm{TW}, \mathrm{HA}$ and $\mathrm{JH}$ contributed significantly to analysis and manuscript preparation; $\mathrm{CH}, \mathrm{TW}$ and $\mathrm{KH}$ performed the data analyses and wrote the manuscript; $\mathrm{CD}$ and $\mathrm{HC}$ helped perform the analysis with constructive discussions

\section{Acknowledgement:}

We acknowledge Ying Hong for contributing to the manuscript. 


\section{References}

1. Holdsworth FW. Fractures, dislocations and fracture-dislocations of the spine. J Bone Joint Surg Am .1970;52:1534-51.

2. Magerl F, Aebi M, Gertzbein SD, et al. A comprehensive classification of thoracic and lumbar injuries. Eur Spine J.1994;3:184-201.

3. Wang BY, Wu TK, Liu H, et al. Biomechanical Analysis of Bilateral Facet Joint Stabilization Using Bioderived Tendon for Posterior Cervical Spine Motion Reservation in Goats. World Neurosurgery. 2017; 107:268-275.

4. Wood KB, Li W, Lebl DR, Ploumis A. Management of thoracolumbar spine fractures. Spine J. 2014 Jan;14(1):145-64.

5. Dahdaleh NS, Viljoen SV , Dalm BD, et al. Posterior ligamentous complex healing following disruption in thoracolumbar fractures. Med Hypotheses. 2013; 81(1):117-118.

6. Jiang T, Ren XJ, Wang WD, et al. Operative treatment for cervical fracture and dislocation with blunt unilateral vertebral artery injury. Chin J Traumatol. 2010;13:279-283.

7. Bostman OM. Absorbable implants for the fixation of fractures. J Bone Joint Surg Am. 1991;73: 148153.

8. Shono Y, Kaneda K, Abumi K,et al. Stability of posterior spinal instrumentation and its effects on adjacent motion segments in the lumbosacral spine. Spine (Phila Pa 1976). 1998;23:1550-1558.

9. Goel VK, Lim TH, Gwon J, et al. Effects of rigidity of an internal fixation device. A comprehensive biomechanical investigation. Spine (Phila Pa 1976).1991;16:S155-S161.

10. McAfee PC, Farey ID, Sutterlin CE, et al. The effect of spinal implant rigidity on vertebral bone density: A canine model. Spine (Phila Pa 1976). 1991;16: S190-S197.

11. Scheer JK, Tang JA, Deviren V, et al. Biomechanical analysis of cervicothoracic junction osteotomy in cadaveric model of ankylosing spondylitis: effect of rod material and diameter. J Neurosurg Spine. 2011;14:330-335.

12. Soderlund $\mathrm{CH}$, Pointillart $\mathrm{V}$, Pedram $\mathrm{M}$, et al. Radiolucent cage for cervical vertebral reconstruction: a prospective study of 17 cases with 2-year minimum follow-up. Eur Spine J. 2004;13:685-690.

13. Kanayama M, Cunningham BW, Haggerty CJ, et al. In vitro biomechanical investigation of the stability and stress-shielding effect of lumbar interbody fusion devices. J Neurosurg. 2000;93:259265.

14. Kanayama M, Cunningham BW, Weis JC, et al. Maturation of the posterolateral spinal fusion and its effect on load-sharing of spinal instrumentation: An in vivo sheep model. $J$ Bone Joint Surg Am. 1997;79: 1710-1720.

15. Schnake K J , Schaeren S, Jeanneret B . Dynamic Stabilization in Addition to Decompression for Lumbar Spinal Stenosis with Degenerative Spondylolisthesis. Spine (Phila Pa 1976). 2006;31: 442449 . 
16. Noyes FR, Barber-Westin SD. Anterior cruciate ligament graft placement recommendations and bonepatellar tendon-bone graft indications to restore knee stability. Instr Course Lect. 2011;60: 499-521.

17. Schlegel TF, Hawkins RJ, Lewis CW, et al. An in vivo comparison of the modifified Mason Allen suture technique versus an inclined horizontal mattress suture technique with regard to tendon-to-bone healing: a biomechanical and histologic study in sheep. J Shoulder Elbow Surg. 2007;16:115-121.

18. Yasuda K, Kitamura N, Kondo E, et al. One-stage anatomic double-bundle anterior and posterior cruciate ligament reconstruction using the autogenous hamstring tendons. Knee Surg Sports Traumatol Arthrosc. 2009; 17(7):800-805.

19. Yonenobu K, Abumi K, Nagata K, Taketomi E, Ueyama K. Interobserver and intraobserver reliability of the japanese orthopaedic association scoring system for evaluation of cervical compression myelopathy. Spine (Phila Pa 1976). 2001 Sep 1;26(17):1890-4; discussion 1895.

20. Vernon H, Mior S. The Neck Disability Index: A study of reliability and validity. J Manipulative Physiol Ther. 1991;14:409-15.

21. Kirshblum SC, Burns SP, Biering-Sorensen F, et al. International standards for neurological classification of spinal cord injury (revised 2011). J Spinal Cord Med. 2011;34(6):535-46.

22. Pavese C, Bachmann L M , Schubert M , et al. Bowel Outcome Prediction After Traumatic Spinal Cord Injury: Longitudinal Cohort Study. Neurorehabilitation and neural repair. 2019; 33(1):154596831986872.

23. Wu CC, Jin HM , Yan YZ, et al. Biomechanical Role of the Thoracolumbar Ligaments of the Posterior Ligamentous Complex: A Finite Element Study. World Neurosurgery, 2018:S1878875018300263.

24. Merter A, Karaca MO , Tarik Yazar. Biomechanical effects of sequential resection of the posterior ligamentous complex on intradiscal pressure and resistance to compression forces. acta orthopaedica et traumatologica turcica. 2019; 53(6).

25. Alapan Y, Demir C, Kaner T, et al. Instantaneous center of rotation behavior of the lumbar spine with ligament failure: Laboratory investigation. J Neurosurg: Spine. 2013;18:617-626.

26. Heuer F, Schmidt H, Klezl Z, et al. Stepwise reduction of functional spinal structures increase range of motion and change lordosis angle. J biomech. 2007;409(2):271-280.

27. Gillespie KA, Dickey JP. Biomechanical role of lumbar spine ligaments in flexion and extension: determination using a parallel linkage robot and a porcine model. Spine. 2004;29:1208-1216.

28. Koller $\mathrm{H}$, Hempfifing A, Acosta F, et al. Cervical anterior transpedicular screw fifixation. Part l: study on morphological feasibility, indications, and technical prerequisites. Eur Spine J. 2008;17:523-38.

29. Koller H, Acosta F, Tauber M, et al. Cervical anterior transpedicular screw fixation (ATPS) - Part II.Accuracy of manual insertion and pull-out strength of ATPS. Eur Spine J. 2008;17:539-55.

30. Koller H, Hitzl W, Acosta F, et al. In vitro study of accuracy of cervical pedicle screw insertion using an electronic conductivity device (ATPS part III). Eur Spine J.2009;18:1300-13.

31. Aramomi M, Masaki Y, Koshizuka S, et al. Anterior pedicle screw fifixation for multilevel cervical corpectomy and spinal fusion. Acta Neurochir (Wien). 2008;150:575-82 discussion 582. 
32. Koller $\mathrm{H}$, Schmidt $\mathrm{R}$, Mayer $\mathrm{M}$, et al. The stabilizing potential of anterior, posterior and combined techniques for the reconstruction of a 2-level cervical corpectomy model: biomechanical study and first results of ATPS prototyping. Eur Spine J. 2010;19:2137-48.

33. Zhao L, Li G, Liu J, et al. Radio logical studies on the best entry point and trajectory of anterior cervical pedicle screw in the lower cervical spine. Eur Spine J. 2014;23:2175-81.

34. Lv GH, Wang B, Kang YJ, et al. Combined anterior and posterior surgery for treatment of cervical fracture-dislocation in patients with ankylosing spondylitis. Chin J Traumatol. 2009;12:148-152

35. Chen JM , Hu Y, Gu YJ , et al. Clinical analysis of one-stage posterior-anterior operative approach in treating lower cervical spine fracture and dislocation]. Chin J Orthop \& Traumatol. 2010; 23(12):93841.

36. Kirkham, B, Wood, et al. Management of thoracolumbar spine fractures. The Spine J. 2014;14(1):145164.

37. Li ZH, Wu HR, Chu J, et al. Motion analysis of dynamic cervical implant stabilization versus anterior discectomy and fusion: a retrospective analysis of 70 cases. Eur Spine J. 2018; 27.

38. Ngo LM , Aizawa $T$, Hoshikawa $T$, et al. Fracture and contralateral dislocation of the twin facet joints of the lower cervical spine. Eur Spine J. 2011; 21(2):282-288.

39. Hamoud K, Hershkovitz I, Hanani A, Marom L, Abbas J. Internal stabilization of a flexion-distraction injury of the upper cervical spine of a toddler: a new technique and literature review. Spine (Phila Pa 1976). 2012 Mar 15;37(6):E400-7.

40. Alexander RV, John DK, Kris ER, et al. AOSpine subaxial cervical spine injury classification system. Eur Spine J. 2016;25(7):2173-2184.

\section{Table}

Table 1 not available with this version

\section{Figures}



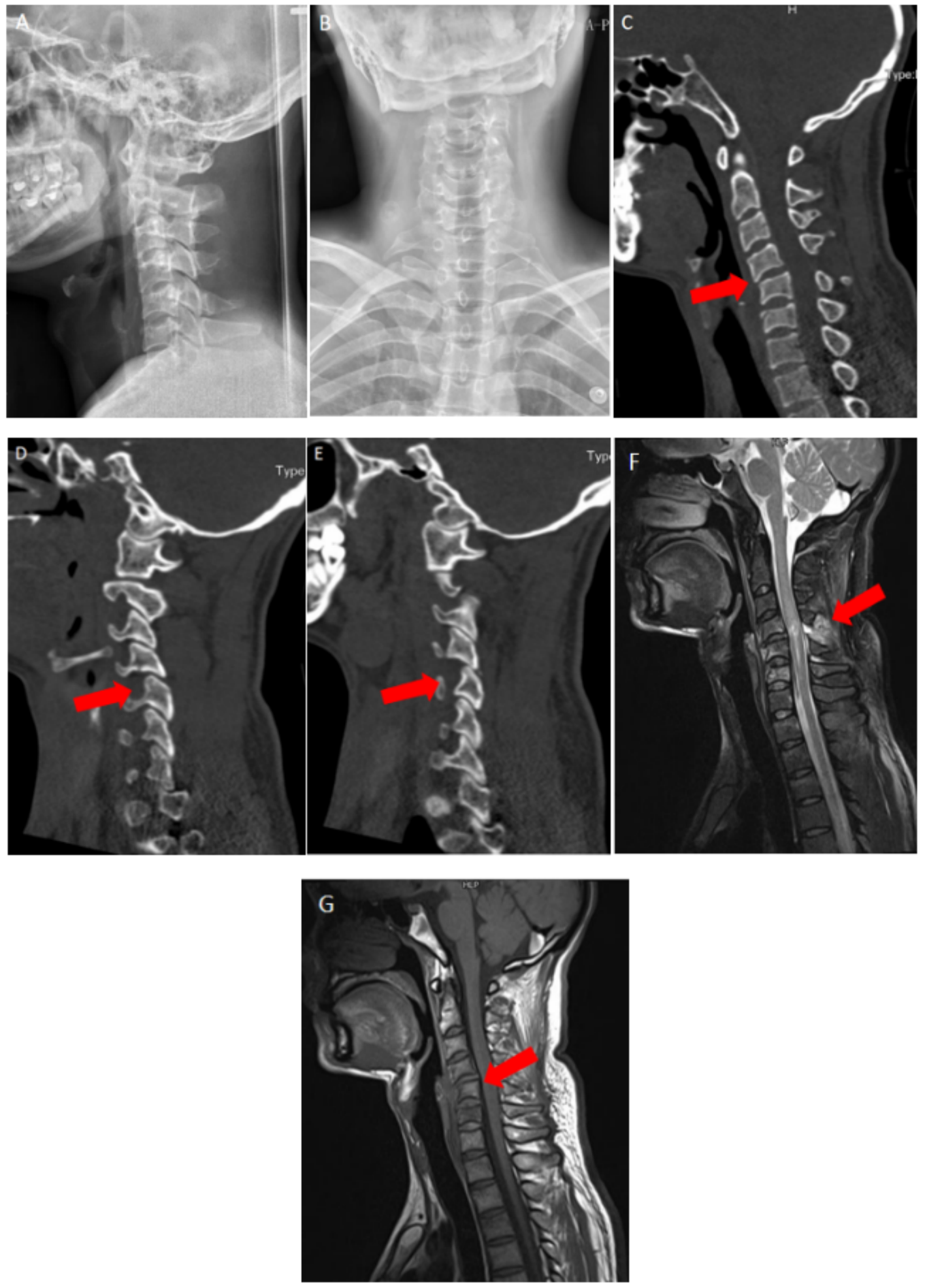

\section{Figure 1}

Preoperative imaging data of the patient. (A\&B) Anteroposterior and lateral radiograph of the cervical spine on admission showing dislocation of C4-6, with overdistraction. (C-E) Sagittal CT images showing dislocation of C4-6 vertebral bodies(C) and bilateral facet joints(D: Left, E: Right); also revealing no damage to the cervical spine bones. $(F, G)$ Sagittal T1-weighted MRI(F) showing no cervical disc 
damage,T2-weighted MRI(G) showing soft tissue edema around cervical the spinous process and spinal cord edema of C4-6, suggesting posterior tension band and spinal cord injury (red arrow).
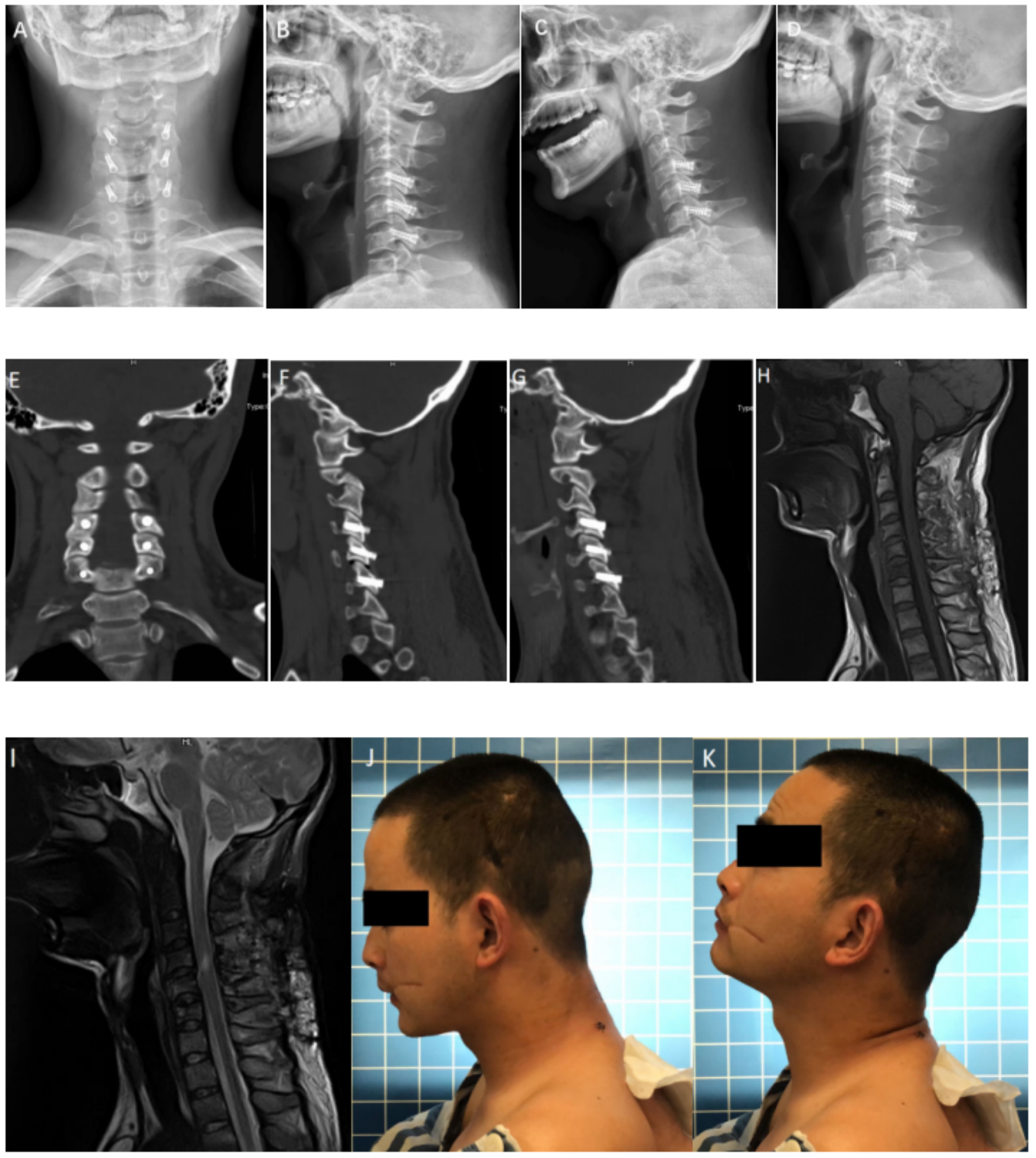

\section{Figure 2}

Imaging data of the patient three days after surgery. (A\&B) Anteroposterior and lateral X-ray radiographs of the cervical spine on admission showing reset C4-6 and restored sagittal contour.(C\&D)Fexion and extension images of cervical spine showing recovery of mobility and stability. (E-G) Sagittal and coronal 
$\mathrm{CT}$ images showing the recovery of the alignment of C4-6 vertebral bodies and bilateral facet joints(F: Left, G: Right). Proper locations of anchoring screws are also confirmed. (H\&I) MRI revealing satisfactory spinal canal decompression and cervical spine sequence. (J\&K) Representative photographs showing the patient's neck postures postsurgery. The neck mobility had significantly improved.
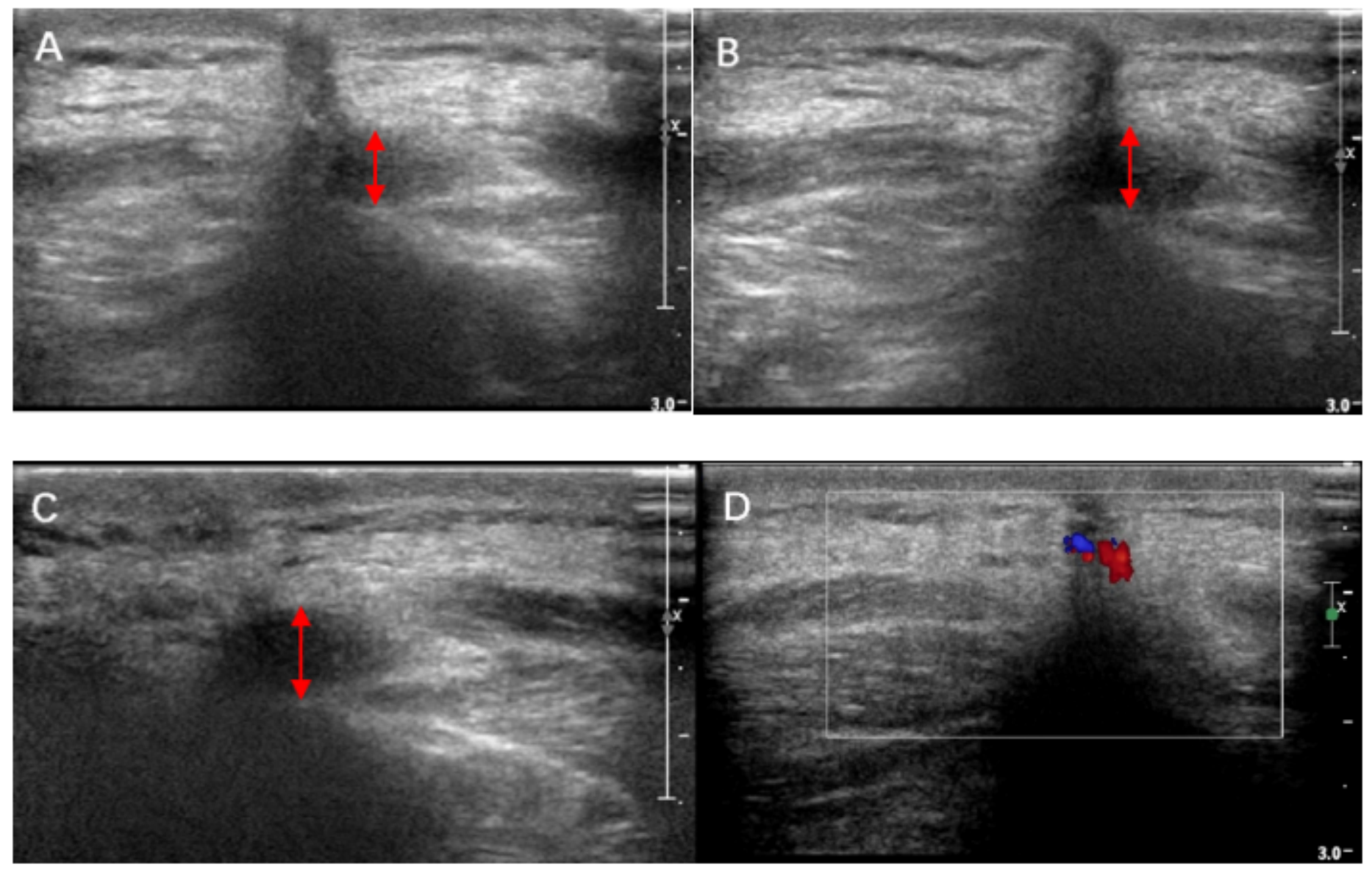

\section{Figure 3}

The color Doppler ultrasound showing satisfactory mechanical properties and peripheral blood supply around the artificial ligament during flexion and extension. (A) Flexion position; the diameter of the artificial ligament was approximately $4.1 \mathrm{~mm}$ (double-headed arrow). (B) Neutral position; the diameter of the artificial ligament was approximately $4.4 \mathrm{~mm}$ (double-headed arrow). (C) Extension position; the diameter of the artificial ligament was approximately $6.5 \mathrm{~mm}$ (double-headed arrow). (D) The peripheral blood supply around artificial ligament. 

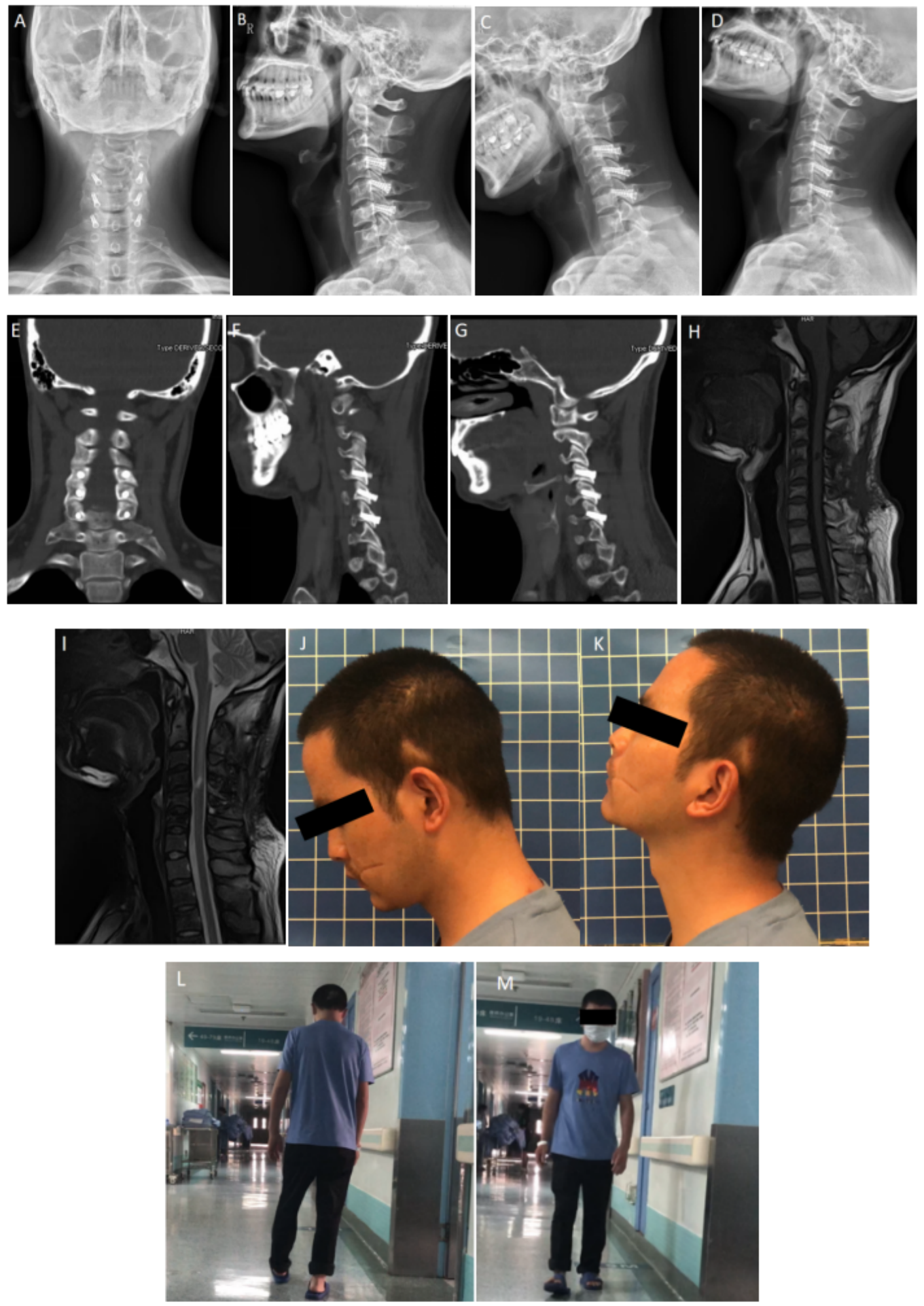

\section{Figure 4}

Imaging data of the patient three months after surgery. (A \& B) Anteroposterior and lateral X-ray radiographs of the cervical spine showed the normal sequence and curvature of the cervical spine and endplates in a normal spinal alignment.(C\&D)Fexion and extension images of cervical spine showing further improvement of mobility and stability. (E-G) Sagittal and coronal CT images showing maintenance of alignment of C4-6 vertebral bodies and facet joints. There was no anchoring screws 
displacement. (H \& I) MRI demonstrating satisfactory healing of the spinal cord and back soft tissue edema. (J-M) Representative photographs of the patient's neck postures after 3-month rehabilitation. The mobility and motor function had further improved.
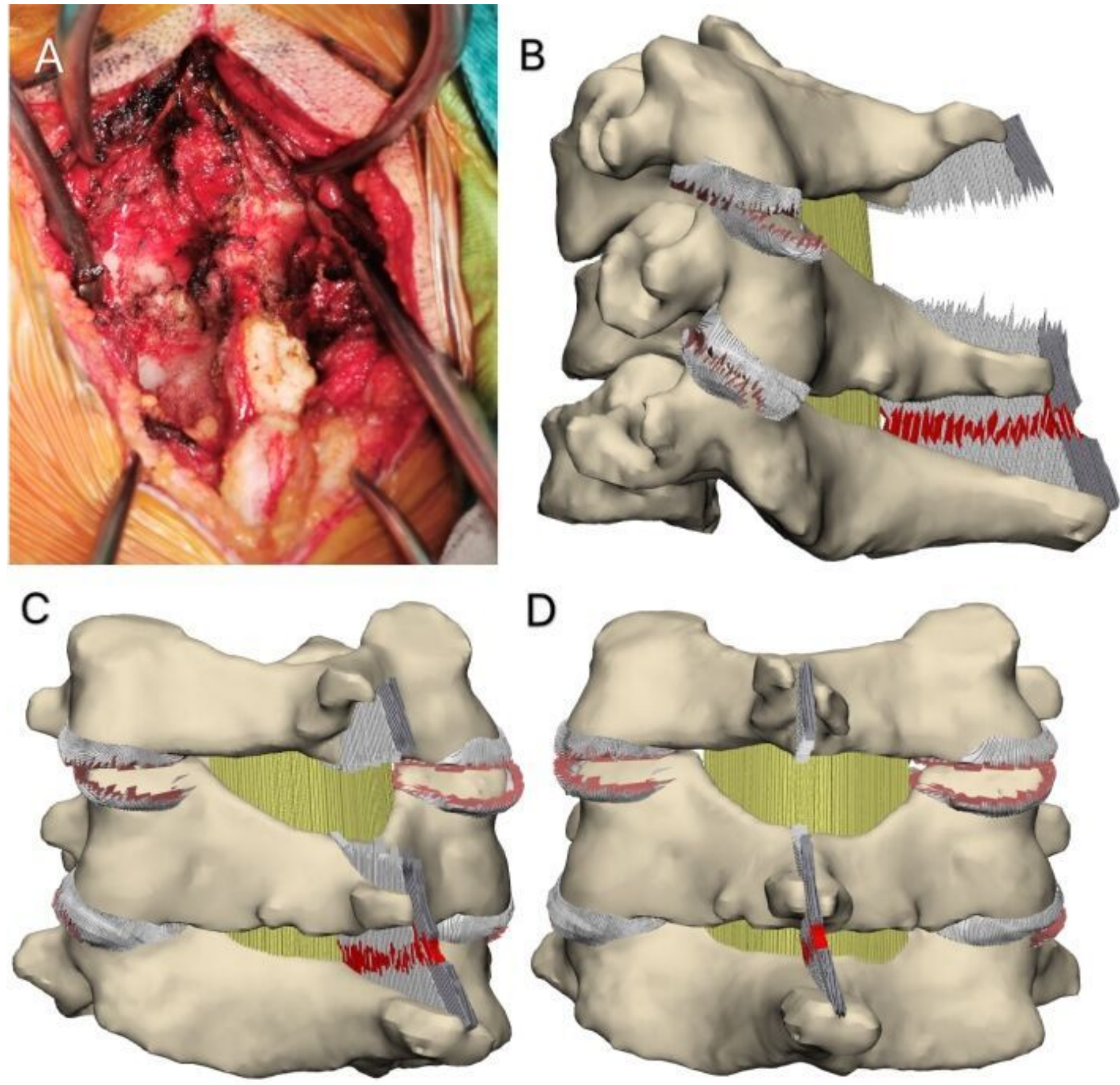

Figure 5

The patient's PLC injury before the surgery. (A) Intraoperative image. Three-dimension (3D) reconstruction diagram. (B) Lateral view; (C) oblique view; and (D) top view. 

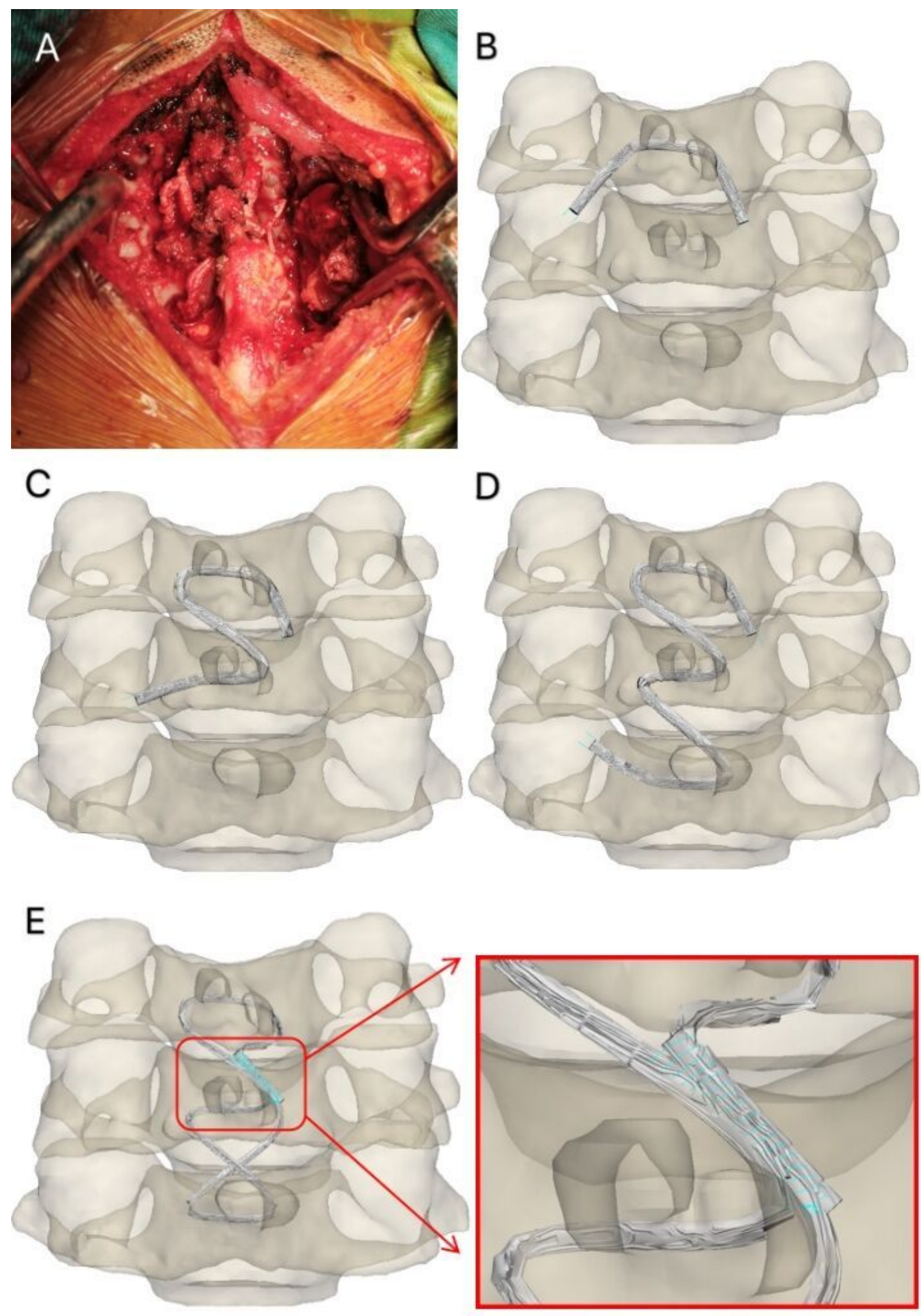

Figure 6

Procedures of C4-6 artificial ligament reconstruction between spinous processes. (A) Intraoperative image. (B-E) Systematic 3D reconstruction from top view images. 

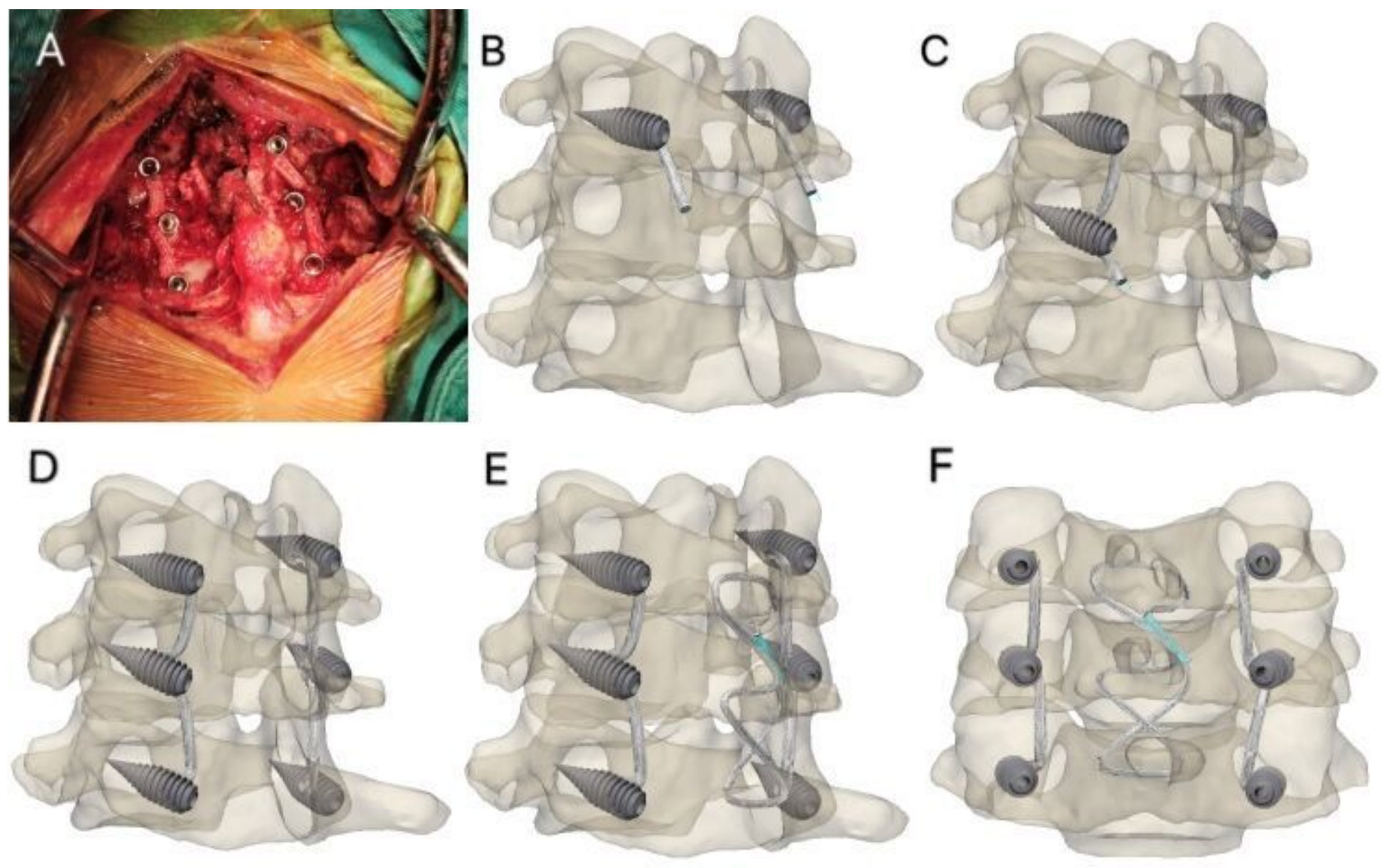

Figure 7

Procedures of C4-6 facet joint artificial ligament anchoring. (A) Intraoperative image. (B-D) 3D reconstruction diagrams in oblique view. (E \& F) The completed state of ligament reconstruction. 